Provided for non-commercial research and education use. Not for reproduction, distribution or commercial use.

ISBN 978-90-481-2890-7

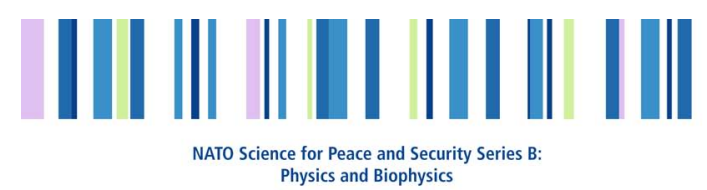

\title{
Properties and Applications of Thermoelectric Materials
}

The Search for New Materials

for Thermoelectric Devices

Edited by

Veljko Zlatic

Alex C. Hewson

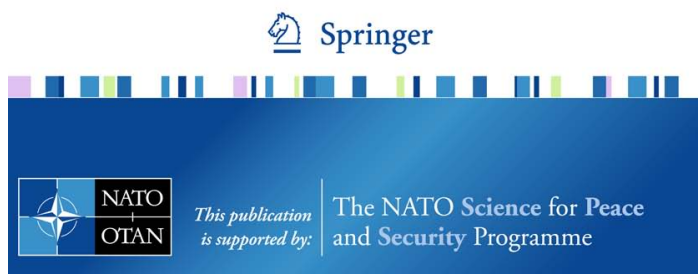

This chapter was published in the above Springer book. The attached copy is furnished to the author for non-commercial research and education use, including for instruction at the author's institution, sharing with colleagues and providing to institution administration.

Other uses, including reproduction and distribution, or selling or licensing copies, or posting to personal, institutional or third party websites are prohibited.

In most cases authors are permitted to post their version of the chapter (e.g. in Word or TEX form) to their personal website or institutional repository. 


\title{
Author's personal copy
}

\section{Thermoelectrics Near the Mott Localization-Delocalization Transition}

\author{
K. Haule and G. Kotliar
}

\section{Introduction}

\subsection{Weakly Correlated Systems}

The dream of accelerating the discovery of materials with useful properties using computation and theory is quite old, but actual implementations of this idea is recent. Successes in material design using weakly correlated materials, are due, to a large degree, to a two important developments:

1. Approximate implementations of the first principles density functional theory, which are relatively accurate and computationally efficient

2. Robust implementation of algorithms which are highly reproducible and widely available

Density functional theory based approaches gives reliable estimates of the total energy, and are an excellent starting point for computing excited state properties of weakly correlated electron systems. These approaches allows the evaluation of transport coefficients using very limited, or no empirical information, and are beginning to be used in conjunction with data mining technique and combinatorial searches.

\subsection{Strongly Correlated Electron Systems}

Since a large number of interesting physical phenomena, such as high temperature superconductivity and large Seebeck coefficients, are realized in strongly correlated materials, there is a great interest in the possibility of carrying out rational material design with correlated materials.

K. Haule and G. Kotliar

Physics Department and Center for Materials Theory, Rutgers University,

136 Frelinghuysen Road, Piscataway, NJ

e-mail: haule@physics.rutgers.edu, kotliar@physics.rutgers.edu

V. Zlatić and A. C. Hewson (eds.), Properties and Applications of Thermoelectric Materials, 119 NATO Science for Peace and Security Series B: Physics and Biophysics,

(c) Springer Science+Business Media B.V. 2009 


\section{Author's personal copy}

The theoretical situation in this area, however, is a lot more uncertain. For example, the issue of whether the two dimensional one band Hubbard model supports superconductivity or not is still very open [10]. Given that this model is an extraordinary oversimplification of realistic materials, it is hard to contemplate explaining, let alone predicting experimental results in materials that require a much more elaborate models for their description. The prospect of predicting properties of materials which have not yet been synthesized is even more daunting. In this chapter we will argue that this assessment is overly pessimistic, and we will give some reasons why we expect a rapid progress in the coming years through the interplay of qualitative reasoning, new theoretical methods, and experiments. We will then describe some attempts in gaining experience in this field, and the lessons that we have learned in the process using thermoelectric performance as an example.

\subsubsection{Dynamical Mean Field Theory}

The advent of Dynamical Mean Field Theory (DMFT) removed many difficulties of the traditional electronic structure methods. DMFT describes Mott insulators, as well as correlated metals. It treats quasiparticle bands and Hubbard bands on the same footing, and, unlike simpler approaches such as LDA+U, is able to describe the multiplet structure of correlated solids. The latter is being inherited from open shell atoms and ions. DMFT has been successful in accounting for the behavior observed in correlated materials ranging from plutonium to vanadium oxides and has even made some predictions, which have been confirmed by experiment. This suggests that the approach is reasonably accurate, in the sense that it gives a zeroth orderpicture of correlated materials, not too close to criticality. Ten years ago, a combination of DMFT with electronic structure methods, LDA+DMFT, wasproposed $[1,8,15]$ and accurate implementations are being actively developed across the world. Just like LDA, these tools connect the atomic positions with the physical observables using very little information from experiment, and therefore they have the potential to accelerate material discovery.

Predicting the phase diagram of strongly correlated materials is an extremely difficult problem. Correlated materials have many competing phases, which are very close in energy. This poses serious difficulties to traditional many body approaches. (i) Terms in the Hamiltonian, present in the actual material, but absent in the model Hamiltonian, can exchange the stability of two very different phases. (ii) Finite size effects or boundary conditions can artificially stabilize a phase, which is not stable in experiment.

DMFT divides the solution of the many-body problem of a solid state system into two separate distinct steps. Common to many mean field approaches, a given Hamiltonian can have many distinct DMFT solutions, describing various possible phases of a material. Which phase is realized for a given value of parameters (temperature, volume, stress, doping concentration of impurities, etc.) is determined by comparing the free energy of the different DMFT solutions. A lot of important information can be obtained from the first step alone, when combined withexperimental information. 


\section{Author's personal copy}

If one knows that for some value of parameters certain phase is realized in material, one can use DMFT to explore the properties of that phase, and optimize desired physical property, side stepping the difficult issue of the comparing the free energies of competing phases. The free energy difference can be computed at a later stage.

\section{The Process of Rational Material Design}

Figure 1 describes schematically the rational material design process. It begins with a qualitative idea, which is then tested by a calculation. One of the major advances of realistic DMFT implementations such as LDA+DMFT or GW+DMFT is that now this calculation can be made material specific, resulting in a set of predictions that can be tested experimentally. The experimental results can either rule out the qualitative idea, in which case the process stops, or reinforce and refine the idea. Experiments also help to calibrate the computational methods, which in turn lead to an improved material specific prediction in the next iteration. Not only materials with improved properties $M_{1}, M_{2}, M_{3}$, result from this approach, but in addition, this process tests theoretical ideas in an unbiased way, deepens our understanding of materials physics, and refines the accuracy of computational tools. Large databases of existing materials are created (e.g. http://icsd.ill.eu/icsd/index.html), which are starting to be used, in combination with the first principle methods, for data mining techniques. Using the crystal structure information from the database, the first principles methods can identify potentially promising materials, which can then be analyzed experimentally.

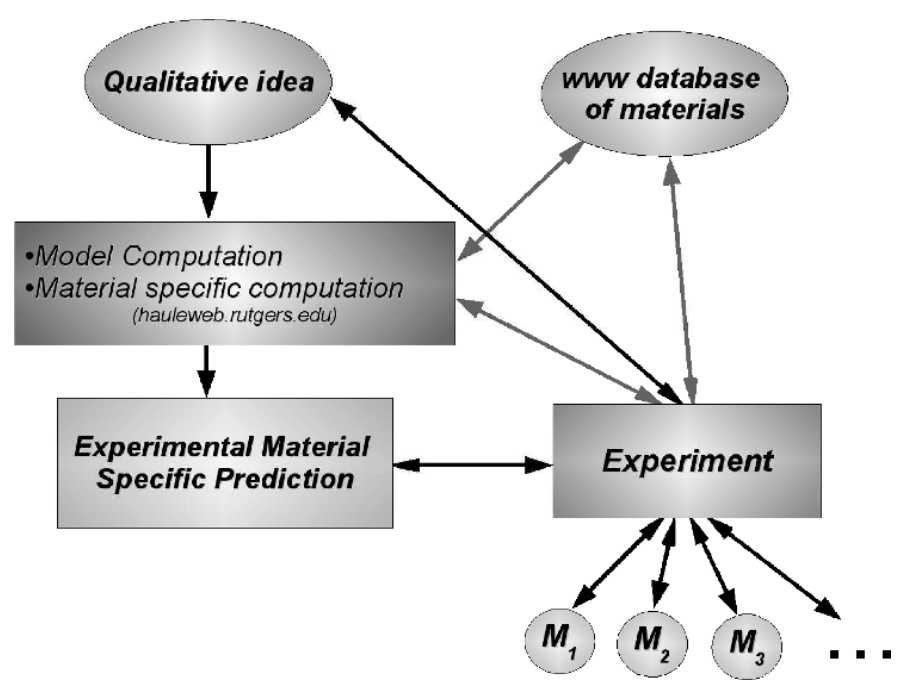

Fig. 1 A schematic drawing of the rational material design process. It relies on condensed matter theory, material databases and realistic DMFT implementations, and it involves a close and iterative interplay of theory and experiment. 


\section{Author's personal copy}

\section{Thermoelectricity of Correlated Materials}

\subsection{Formalism}

The transport coefficients that govern the thermopower, electric and thermal conductivity can be expressed in terms of the matrix of kinetic coefficients $A_{m}$ relating the electric and thermal currents $J, J_{Q}$ to the applied external fields $\nabla \mu / T, \nabla T / T^{2}$. Transport quantities become $S=-\left(k_{B} / e\right)\left(A_{1} / A_{0}\right), \sigma=\left(e^{2} / T\right) A_{0}, \kappa=k_{B}^{2}\left[A_{2}-\right.$ $\left.A_{1}^{2} / A_{0}\right]$. The thermoelectric response thus reduces to the evaluation of kinetic coefficients.

The thermoelectric figure of merit is defined by

$$
Z T=\frac{S^{2} \sigma T}{\kappa+\kappa_{\text {phonon }}},
$$

where $T$ is the absolute temperature, $\sigma$ is the electrical conductivity, $S$ is the Seebeck coefficient or thermopower, and $\kappa\left(\kappa_{\text {phonon }}\right)$ is the electron (phonon) contribution to the thermal conductivity.

The Wiedemann-Franz law is an approximate relation that allows us to estimate the ratio of the electronic contribution to the thermal conductivity $(\kappa)$ and electric conductivity $(\sigma)$. It postulates that the Lorentz number, $L=\kappa /(\sigma T)$, is weakly material dependent.

Its value at low temperatures is given by $\left(\pi^{2} / 3\right)\left(k_{B} / e\right)^{2}=2.44 \times 10^{-8} \mathrm{~W} \Omega / K^{2}$. We will return to the Lorentz number at higher temperatures later in this article. If we ignore the thermal conductivity of the lattice, the figure of merit can be written as $Z T=S^{2} / L$, hence to have a promising figure of merit (ZT close to or larger than one) it is necessary to have $S$ bigger than the basic scale $k / e=86 \times 10^{6} \mathrm{~V} / \mathrm{K}$. The thermal current of an interacting electronic system was determined first by Mahan and Jonson [11]. Reference [11] discusses a model containing electrons interacting with phonons, and the review [16] discusses the general case of the electron-electron interactions (see also Ref. [22]).

DMFT expresses the one particle Greens function in terms of a local self energy of an impurity model, satisfying a self consistency condition. Practical evaluation of the transport coefficients becomes possible in the approximation of small vertex corrections. This was first done by Schweitzer and Czycholl [25] (see also Ref. [23]). For the Hubbard-like interactions, there are no contributions from the nonlocal Coulomb interactions, and the neglect of the vertex corrections can be justified rigorously in the limit of infinite dimensions [13]. The same is true, but far less obvious, for the thermal current, as it was shown in Ref. [22]. In the multi-orbital situation, the vertex corrections to the conductivity need to be examined on a case by case basis, and do not necessarily vanish, even in infinite dimensions. With this approximation, the LDA+DMFT transport coefficients reduce to

$$
A_{m}^{\mu v}=\pi T \int d \omega\left(-\frac{d f}{d \omega}\right)\left(\frac{\omega}{T}\right)^{m} \sum_{\mathbf{k}} \operatorname{Tr}\left[v_{\mathbf{k}}^{\mu}(\omega) \rho_{\mathbf{k}}(\omega) v_{\mathbf{k}}^{v}(\omega) \rho_{\mathbf{k}}(\omega)\right]
$$




\section{Author's personal copy}

Thermoelectrics Near the Mott Localization-Delocalization Transition

where $v_{\mathbf{k}}^{\mu}=-i \int d \mathbf{r} e^{-i \mathbf{k r}} \frac{\partial}{\partial x^{\mu}}$ are velocities of electrons and $\rho_{\mathbf{k}}$ is the electron spectral density

$$
\rho_{\mathbf{k}}(\omega)=\frac{1}{2 \pi i}\left[G_{\mathbf{k}}^{\dagger}(\omega)-G_{\mathbf{k}}(\omega)\right]
$$

The weakly interacting case appears as a limiting case where the spectral function becomes a delta function $\rho_{\mathbf{k}}(\varepsilon)=\sum_{i} \delta\left(\varepsilon-\varepsilon_{\mathbf{k} i}\right)$. One can therefore formulate the problem of the optimization of the figure of merit as the problem of optimizing a functional of spectral functions, with self energies which are realizable from an Anderson impurity model, with a bath satisfying the DMFT self-consistency condition.

\subsection{Thermoelectricity near the Mott Transition: Qualitative Considerations}

Following the early developments of DMFT and its successful application to the theory of the Mott transition in three dimensional transition metal oxides [6], it was natural to use this approach to formulate and answer the question of whether we should look for good thermoelectrics near the Mott localization-delocalization transition. The theoretical answer to this central issue of this article is no, but perhaps yes.

There were several reasons to suspect that proximity to the localizationdelocalization transition is good for thermoelectricity:

1. Sharp structures in the density of states lead to large $S$ in simple theories [17]. The modern theory of the Mott transition predicts a quasiparticle peak, which narrows as the transition is approached. And this could result in a large thermoelectric response.

2. One can think on a qualitative level of the thermoelectric coefficient as the entropy per carrier. In the incoherent regime, one could imagine that each carrier can transport a large amount of entropy. The incoherent regime, above a characteristic coherence temperature $T^{*}$, is easy to access near a localizationdelocalization transition, because the proximity to this boundary makes $T^{*}$ low.

3. Orbital degeneracy increases the number of carriers and would be expected to increase the figure of merit. There are many orbitally degenerate three dimensional correlated transition metal oxides.

Reference [14] considered a model of the prototypical doped insulator $\mathrm{LaSrTiO}_{3}$, which has been carefully investigated in a series of papers [26]. The thermoelectric properties of this system had not been investigated at that time. Early DMFT studies accounted for the divergence of the linear term of the specific heat, and the susceptibility, as well as the existence of a quasiparticle peak in the spectra [28].

The Hall coefficient, however, coincides with the band theory calculations, and is non-critical near the Mott transition [12]. It is possible to analyze the DMFT transport equations in two regimes: (i) $T \ll T^{*}$, where the electronic transport is 


\section{Author's personal copy}

controlled by band-like coherent quasiparticles, well described in momentum space, (ii) $T \gg T^{*}$ when the electron is better described as a particle in real space, and the transport is diffusive [14] (see below). The second regime is well described by the high temperature expansion, valid for $T>D$ ( $D$ is the bandwidth), and by comparison with approximate numerical solutions of the DMFT equations. In Ref. [14] it was noticed that the thermoelectric response of the high temperature regime matches smoothly with the low response at low temperatures, valid for $T \ll T^{*}$.

\subsection{Application to $\mathrm{LaSrTiO}_{3}$}

An approximate numerical solution of the DMFT equations for the titanides was shown to interpolate smoothly between the high temperature and low temperature region. This is consistent with the idea that DMFT reconciles the band picture at low energies and low temperatures, with the particle picture at high energies and high temperatures. The temperature scale here is set by the coherence temperature $T^{*}$. Taking a tight binding parametrization suitable for the titanites, the figure of merit as a function of temperature and doping is reproduced in Fig. 2.

The behavior of the thermoelectric power near the Mott transition is shown in Fig. 3. Notice that at low doping, the contribution from the lower Hubbard

Fig. 2 Figure of merit for different values of the lattice thermal conductivity.
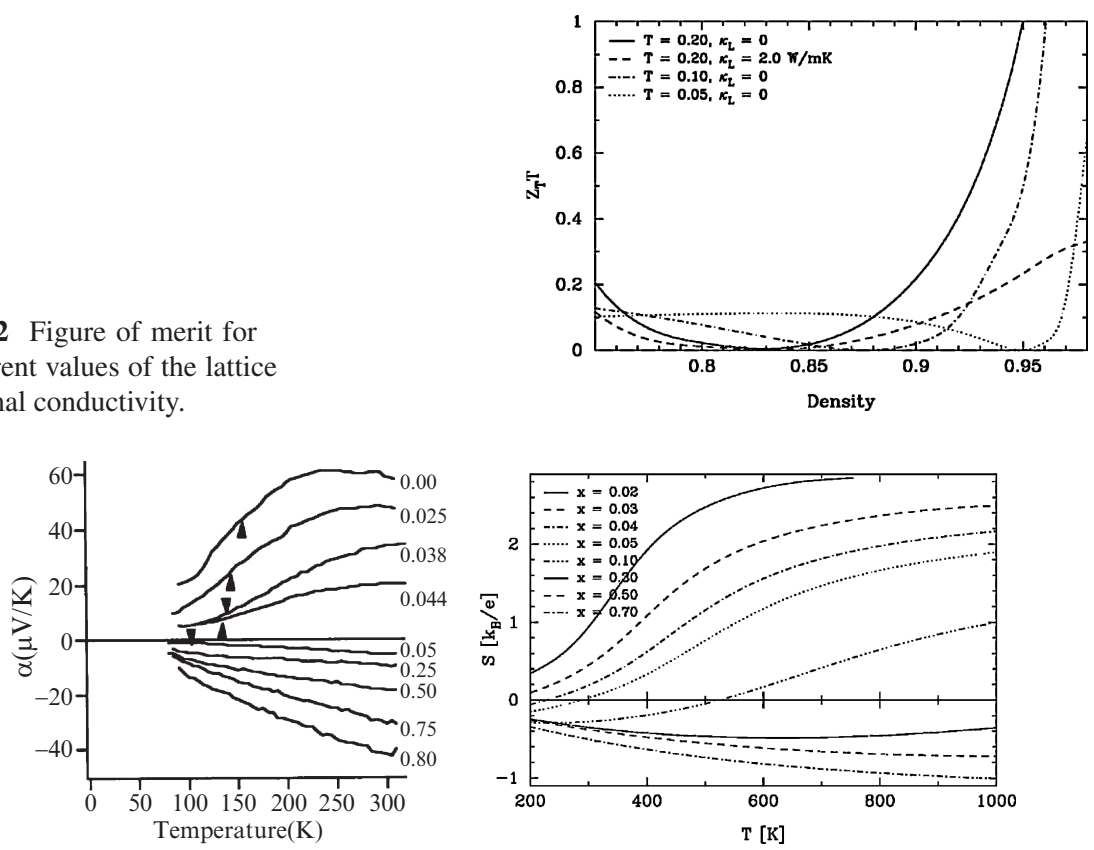

Fig. 3 Experimental (left panel) and theoretical computations of the thermoelectric power of the titanites from Refs. [7, 14]. 


\section{Author's personal copy}

band dominates and the thermoelectric power is positive while at high doping the quasiparticle contribution dominates and the thermoelectric power is electron-like. Measurements near the Mott transition were carried out a few years later [7], and they are qualitatively, but not quantitatively, similar to the theory. This is to be expected, given the various approximations that were made (the electronic structure, the lattice distortion, and crystal field effects ignored, the impurity solvers used were very approximate).

\subsection{Low Temperature Regime}

$\mathrm{LaSrTiO}_{3}$ is described by a multi-band Hubbard model. At low temperatures, the Fermi liquid theory is valid. The slope of the real part of the self energy scales as $1-1 / Z$, where $Z$ is the quasiparticle residue. The quadratic part of the self energy is related to the quasiparticle lifetime, which is small in the Fermi liquid regime.

Under these assumptions, we can rewrite a simpler expressions for the transport coefficients $A_{n}$ of a multiband Hubbard model at low temperatures

$$
A_{n}^{\mu \nu}=\frac{N k_{B} T}{8} \int_{-\infty}^{\infty} d x \frac{x^{n}}{\cosh ^{2}(x / 2)} \frac{\Phi_{\mu v}\left(x T+\mu-\Sigma^{\prime}(x T)\right)}{\Sigma^{\prime \prime}(x T)},
$$

where $\Phi_{\mu v}$ is the transport function defined by $\Phi_{\mu v}=\sum_{\mathbf{k}} v_{\mathbf{k}}^{\mu} v_{\mathbf{k}}^{v} \delta\left(\omega-\varepsilon_{\mathbf{k}}\right)$ and $\Sigma^{\prime \prime}(\omega)$ is the imaginary part of the electron self-energy.

At low temperatures, $A_{0}$ and $A_{2}$ are simply estimated by replacing $\Sigma^{\prime \prime}(\omega)$ by its quadratic approximation, $\Sigma^{\prime \prime}(\omega) \sim \frac{\gamma_{0}}{Z^{2}}\left(\omega^{2}+\pi^{2} T^{2}\right) \equiv \Sigma^{(2)}(\omega)$. We then obtain

$$
A_{2 n}=\frac{Z^{2}}{T} \frac{N k_{B}}{2 \gamma_{0} \pi^{2}} E_{2 n}^{1} \Phi_{\mu v}\left(\mu_{0}\right)
$$

where $\mu_{0}=\mu-\Sigma^{\prime}(0)$ and

$$
E_{n}^{k}=\int_{-\infty}^{\infty} \frac{x^{n} d x}{4 \cosh ^{2}(x / 2)\left[1+(x / \pi)^{2}\right]^{k}}
$$

are numerical constants of the order unity.

On the other hand, this approximation neglects particle-hole asymmetry and gives zero thermoelectricity since $E_{1}^{1}=0$. There are two sources of particle-hole asymmetry. One is obtained by expanding the transport function in Eq. (4) to first order, which describes the particle-hole asymmetry in the electronic velocities, contained in the bare band structure of the problem. This term can be approximated by the LDA Seebeck coefficient divided by quasiparticle renormalization amplitude $Z$. 


\section{Author's personal copy}

126

K. Haule and G. Kotliar

The second contribution is the result of the particle hole asymmetry of the scattering rate. It involves subleading cubic terms in the self energy, which scale near the Mott transition as

$$
\begin{aligned}
& \Sigma^{\prime \prime}(\omega)=\Sigma^{(2)}(\omega)+\Sigma^{(3)}(\omega)+\cdots, \\
& \Sigma^{3}(\omega)=\frac{\left(a_{1} \omega^{3}+a_{2} \omega T^{2}\right)}{Z^{3}},
\end{aligned}
$$

and $a_{1}, a_{2}$ are constants of order unity (even terms in frequency are not important). This leads to the following expression for the thermoelectric coefficient:

$$
A_{1}=Z \frac{N k_{B}}{2 \gamma_{0} \pi^{2}}\left[\Phi_{\mu v}^{\prime}\left(\mu_{0}\right) E_{2}^{1}-\Phi_{\mu v}\left(\mu_{0}\right)\left(a_{1} E_{4}^{2}+a_{2} E_{2}^{2}\right) / \gamma_{0}\right]
$$

where $\Phi^{\prime}(x)=d \Phi(x) / d x$.

Unfortunately it has proved to be very difficult to estimate the magnitude of the coefficients $a_{1}$ and $a_{2}$. It is important to develop intuition into when these terms are important and their sign. Since in many cases, LDA predicts the correct sign of the thermoelectric power at low temperatures, perhaps the scattering time particle-hole asymmetry Eq. (5) is not dominant in the $\mathrm{LaTiO}_{3}$ system but should be investigated carefully in other materials.

At low temperature, the thermoelectric coefficients is

$$
S=-\frac{k_{B}}{|e|} \frac{k_{B} T}{Z}\left[\frac{\Phi^{\prime}\left(\mu_{0}\right)}{\Phi\left(\mu_{0}\right)} \frac{E_{2}^{1}}{E_{0}^{1}}-\frac{a_{1} E_{4}^{2}+a_{2} E_{2}^{2}}{\gamma_{0} E_{0}^{1}}\right]
$$

which clearly scales as $T / Z$ with $Z$ vanishing at the Mott transition. Since the linear term of the specific heat $\gamma$ scales as $1 / Z$ the ratio $S /(\gamma T)$ in a Hubbard-like model approaches a finite value as $Z$ vanishes:

$$
\frac{S}{\gamma T}=-\frac{3}{|e|} \frac{1}{D\left(\mu_{0}\right)}\left[\frac{\Phi^{\prime}\left(\mu_{0}\right)}{\Phi\left(\mu_{0}\right)} \frac{E_{2}^{1}}{E_{0}^{1}}-\frac{a_{1} E_{4}^{2}+a_{2} E_{2}^{2}}{\gamma_{0} E_{0}^{1}}\right] .
$$

The first part of the ratio depends only on the bare band-structure quantities and is not effected by strong correlations. The second part, however, is due to the asymmetry of the quasiparticle lifetime, and might be less universal and more material and correlation specific. This question deserves further study.

For the $\mathrm{LaSrTiO}_{3}$ system, we estimated its value numerically using LDA+DMFT [21] and we include its value in the plot of Behnia et al. [2] in Fig. 4. In Ref. [2] it was observed that the ratio $S / \gamma T$ is weakly material dependent in a large number of materials which they compiled. From the theoretical point of view, the weak dependence of the ratio of Behnia et al. on material can be view as a validation of the local approximation, since the most material dependence is embodied in the quantity $Z$, which cancels in the ratio $S /(\gamma T)$. This suggest that the DMFT approach holds great promise for the search of good thermoelectric materials. Deviations 


\section{Author's personal copy}

Thermoelectrics Near the Mott Localization-Delocalization Transition

Fig. 4 Bhenia Jaccard

Flouquet plot from Ref. [2].

The theoretical point obtained on the LaSrTiO3 system with $20 \%$ doping away from the Mott insulator is also shown in the same graph.

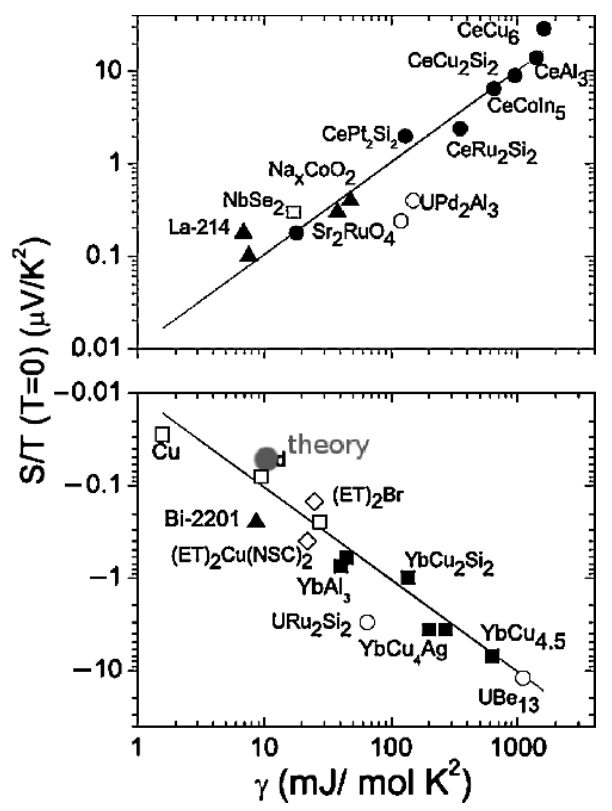

from universality arise from the variations of the bare density of states and from the effects of the cubic terms in the self energy that were not included in the analysis of Ref. [14]. It would be interesting to return to this problem using modern LDA+DMFT tools.

\subsection{High Temperature Results}

In the high temperature region, the expansion of the solution of the DMFT equations led to the celebrated Heikes formula for the Seebeck coefficient. In this limit, thermopower is given by $S=\mu /(e T)$, where $\mu$ is the chemical potential. The exact diagonalization of the atomic problem gives a set of atomic eigenvalues $E_{m}$ and their degeneracies $d_{m}$. The chemical potential is then determined from the partition sum

$$
n=\sum_{m} d_{m} e^{-\beta\left(E_{m}-\mu N\right)} / Z
$$

where $n$ is the number of electrons in a correlated orbital. Hence, the valence of the solid, $n$, can be used to predict the high temperature value of thermopower.

For the case of $n \leq 1$, which is relevant for the titanides, the expressions for transport quantities take the explicit form:

$$
\begin{aligned}
\sigma & =\frac{e^{2}}{a \hbar} \pi N(D \beta) \gamma_{0} \frac{\frac{n}{N}(1-n)}{\left[\frac{n}{N}+\left(1-n^{2}\right)\right]^{2}}, \\
S & =\frac{k_{B}}{e} \log \frac{n}{N(1-n)},
\end{aligned}
$$




\section{Author's personal copy}

128

K. Haule and G. Kotliar

$$
\kappa=\frac{k_{B} D}{a \hbar} \pi N(D \beta)^{2} \gamma_{2} \frac{\frac{n}{N}(1-n)}{\left[\frac{n}{N}+(1-n)\right]^{2}} .
$$

Here $N$ is the spin and orbital degeneracy, and $n$ is the electron density, $D$ is half of the bare bandwidth and $\gamma_{0}, \gamma_{2}$ are numerical constants of order unity.

Notice that at high temperature the Lorentz number is given by $L=(k / e)^{2}(D / k T)^{2}$ $\gamma_{2} / \gamma_{0}$. Hence the Lorentz number in a model with a fixed number of particles and finite bandwidth goes to zero at high temperatures. Thus eventually the electronic thermal conductivity becomes less than the lattice conductivity and the latter controls the figure or merit. This effect was modeled in the dashed curve of Fig. 2, where the effects of the lattice thermal conductivity was modeled by a constant $2.0 \mathrm{~W} / \mathrm{mK}$. The inclusion of the lattice thermal conductivity resulted in a dramatic reduction of the figure of merit. We can interpret the high temperature DMFT results for the thermal transport using a well known equation $\kappa=\frac{1}{3} v_{F} c_{V} l$, where $v_{F}$ is the Fermi velocity, $c_{V}$ the specific heat, and $l$ the electron mean free path. Since the specific heat decreases as $(D / T)^{2}$, the mean free path has saturated to a lattice spacing, and the velocity of the electrons is of the order of $v_{F}$. This is consistent with the value of the conductivity if one uses the Einstein relation $\sigma=D_{c} d n / d \mu$ with $d n / d \mu \approx 1 / T$ and the charge diffusion constant $D_{c}=v_{F} l$. Here the mean free path $l$ is of the order of the lattice spacing, and the Fermi velocity $v_{F}$ is approximately temperature independent.

\section{Towards Material Design}

\subsection{Rules for Good Correlated Thermoelectricity}

From the theoretical analysis it becomes clear why $\mathrm{LaSrTiO}_{3}$ is not a good thermoelectric material. The contributions from the Hubbard bands and the quasiparticle peak have opposite signs, and they compete with each other in the interesting temperature regime, when $T$ is comparable to $T^{*}$. This observation leads to empirical rules for the search for good correlated thermoelectric materials:

1. The optimal performance (when the thermal conductivity of the lattice is taken into account) occurs in the crossover region $T \approx T^{*}$. Hence one should tune $T^{*}$ to the temperature region where the thermoelectric device operates. One should also reduce the electronic thermal conductivity (and therefore also the electric conductivity) until it becomes comparable to the lattice thermal conductivity, but not any further.

2. In the crossover regime, both the quasiparticle bands and the Hubbard bands contribute to the transport. Hence one should try to optimize both high temperature and low temperature expressions for the figure of merit. Therefore good candidates for thermoelectricity have quasiparticle carriers and Hubbard band carriers of the same sign. 


\section{Author's personal copy}

Thermoelectrics Near the Mott Localization-Delocalization Transition

We see that $\mathrm{LaSrTiO}_{3}$ does NOT satisfy the second rule, and hence its figure of merit is not large. The quasiparticle contribution to the thermopower is electron-like while the lower Hubbard band contribution is hole-like.

In contrast, the cobaltates have one hole in the lower Hubbard band, and the quasiparticle contribution evaluated from the LDA [27] has a positive sign, hence it satisfies the second rule for good thermoelectricity (assuming that the contribution from the asymmetry in the scattering rate does not modify the sign of the Seebeck coefficient).

An investigation of the density driven Mott transition in the context of a two band Hubbard model, with one electron per site, was carried out in Ref. [20], and the qualitative analysis is very similar to the doping driven Mott transition.

\subsection{Emergent Mottness}

Interest in thermoelectricity near the doping driven Mott transition leads to theoretical and experimental investigations of $\mathrm{La}_{1-x} \mathrm{Sr}_{x} \mathrm{TiO}_{3}$ and $\mathrm{CoO}_{2} \mathrm{Na}_{x}$ for small values of the concentration parameter $x$. Both theory and experiment suggest that the thermoelectric figure of merit is not very large in this regime. On the other hand, the vicinity of the band insulator end, $\mathrm{La}_{1-x} \mathrm{Sr}_{x} \mathrm{TiO}_{3}$ [19] and $\mathrm{CoO}_{2} \mathrm{Na}_{x}$ (see Fig. 5

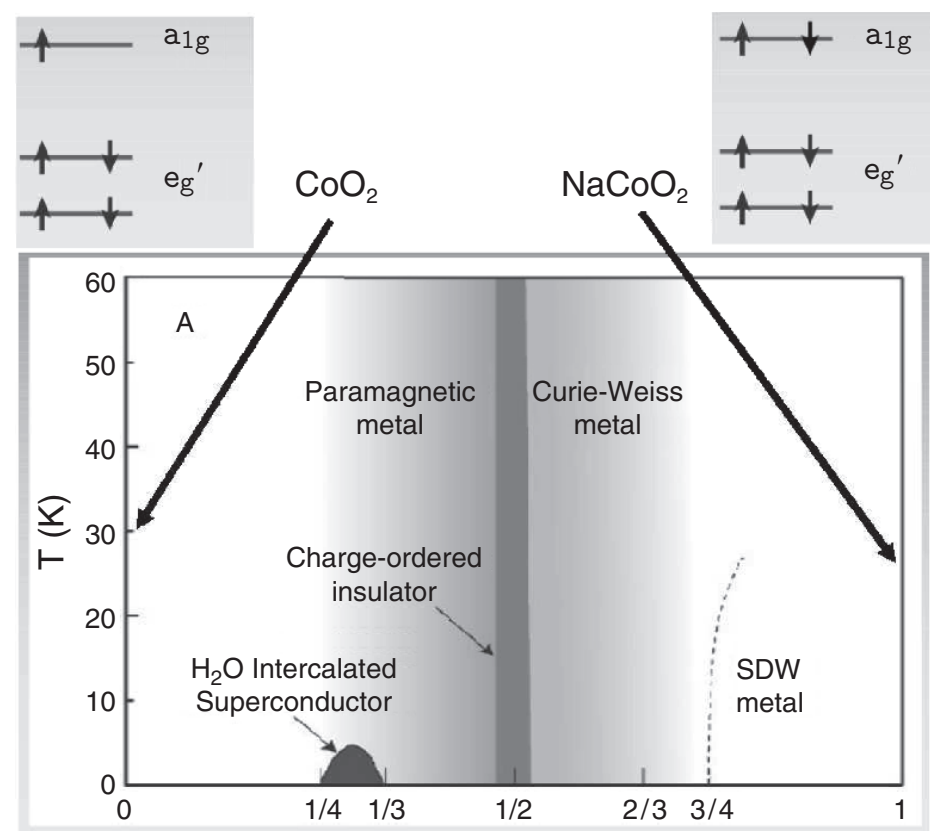

Fig. 5 Phase diagram of $\mathrm{CoO}_{2} \mathrm{Na}_{x}$ compound from Foo et al. [5]. The Mott insulating side at $x=0$ has low thermopower, while the thermopower is greatly enhanced in the vicinity of the band insulator at $x=1$. 


\section{Author's personal copy}

for the phase diagram) were shown to have promising thermoelectric performance. Should we conclude that Mottness is bad for thermoelectricity? Not necessarily, after all, clear signatures of correlation were found in more realistic modeling of doped band insulators, once the impurity potentials of the dopant atoms were taken into account [24]. The impurity potential was found to restrict the spatial regions available for the motion of the electricity and heat carriers. In this restricted configuration space, the occupancy of the electrons is close to integer and Mott physics is realized.

We have suggested that a similar situation occurs in the electron gas close to the metal insulator transition. Here, the long range Coulomb interaction generates short range charge crystalline lattice order. The occupancy of these lattice sites is close to integer filling, suggesting that the character of the metal to insulator transition is that of a Wigner-Mott transition [3]. The mechanism, spatial or orbital differentiation results in a restricted low energy configuration, making Mott physics relevant. This mechanism is quite general, and operates in other materials such as the ruthenates [18]. It could be called emergent Mottness or super-Mottness, and contains similar physics to the orbital selective Mott transition phenomena. Hence (super) Mottness might be relevant for high performance thermoelectricity after all!. It would be useful to reconsider the most recent advances in thermoelectric materials in this light, and investigate the local magnetic susceptibility at the impurity sites of the high performance thermoelectrics $[4,9]$.

\section{Outlook}

The outlook for material design in the field of thermoelectric is quite promising. DMFT seems to capture qualitative trends in oxides of practical interest, furthermore we have simple qualitative ideas, which can be refined and tested with tools of ever increasing precision. In this context, the new thermoelectric modules to be developed in conjunction with the new generation of LDA+DMFT codes, look very appealing. In conjunction with the renewed experimental efforts in this field, the future looks very promising.

Acknowledgements K. Hanle is supported by a grant of the ACS of the Petroleum Research Fund. G. Kotliar is supported by the NSF.

\section{References}

1. Anisimov, V.I., Poteryaev, A., Korontin, M., Anohkin A., and Kotliar, G., J. Phys. Condens. Matter 9, 7359 (1997).

2. Behnia, K., Jaccard, D., and Flouquet, J., Phys.: Condens. Matter 16, 5187 (2004).

3. Camjayi, A., Haule, K., Dobrosavljevic, V., Kotliar, G., Nature Phys. 4, 932 (2008). 


\section{Author's personal copy}

Thermoelectrics Near the Mott Localization-Delocalization Transition

4. Chen, N., Gascoin, F., and Jeffrey Snyder, G., Mueller, E., Karpinski, G., and Stiewe, C., App. Phys. Lett. 87, 171903 (2005).

5. Foo, M.L., Wang, Y., Watauchi, S., Zandbergen, H.W., He, T., Cava, R.J., and Ong, N.P., Phys. Rev. Lett. 92, 247001 (2004).

6. Georges, A., Kotliar, G., Krauth, W., and Rozenberg, M., Rev. of Mod. Phys. 68, 13-125 (1996).

7. Hays, C.C., Zhou, J.-S., Markert, J.T., and Goodenough, J.B., Phys. Rev. B 60, 10367 (1999).

8. Held, K., Adv. Phys. 56, 829 (2007).

9. Hsu, K.F., Loo, S., Guo, F., Chen, W., Dyck, J.S., Uher, C., Hogan, T., Polychroniadis, E.K., and Kanatzidis, M.G., Science 303, 818 (2004).

10. Imai Y., Imada, M., J. Phys. Soc. Japan 75, 094713 (2006).

11. Jonson, M., and Mahan, G. D., Phys. Rev. B, 21, 4223 ( 1980).

12. Kajueter, H., Kotliar, G., and Moeller, G., Phys. Rev. B 53, 16214 (1996).

13. Khurana, A., Phys. Rev. Lett. 64, 1990 (1990).

14. Kotliar, G., and Palsson, G., Phys. Rev. Lett. 80, 4775 (1998).

15. Kotliar, G., Savrasov, S.Y., Haule, K., Oudovenko, V.S., Parcollet, O., and Marianetti, C., Rev. Mod. Phys. 78, 865 (2006).

16. Mahan, G.D., Solid State Phys. 51, 81 (1998).

17. Mahan, G.D., and Sofo, J.O., Proc. Natl. Acad. Sci. U.S.A. 93, 7436 (1996).

18. Neupane, M., Richard, P., Pan, Z.-H., Xu Y., Jin, R., Mandrus, D., Dai, X., Fang, Z., Wang, Z., Ding, H., arXiv:0808.0346.

19. Okuda, T., Nakanishi, K., Miyasaka, S., Tokura, Y., Phys. Rev. B 63, 113104 (2001).

20. Oudovenko, V.S., and Kotliar, G., Phys. Rev. B 65, 075102 (2002).

21. Oudovenko, V.S., Palsson, G., Haule, K., Kotliar, G., and Savrasov, S.Y., Phys. Rev. B 73, 035120 (2006).

22. Paul, I., and Kotliar, G., Phys. Rev. B 67, 115131 (2003).

23. Pruschke, T., Jarrell, M., and Freericks, J., Adv. Phys. 44, 187 (1995).

24. Sarma, D.D., Barman, S.R., Kajueter, H., Kotliar, G., Europhys. Lett. 36, 307 (1996).

25. Schweitzer, H., and Czycholl, G., Phys. Rev. Lett. 67, 3724 (1991).

26. Tokura, Y., Taguchi, Y., Okada, Y., Fujishima, Y., Arima, T., Kumagai, K., Iye, Y., Phys. Rev. Lett. 70, 2126 (1993).

27. Xiang, H.J., and Singh, D.J., Phys. Rev. B 76, 195111 (2007).

28. Yoshida, T., Ino, A., Mizokawa, T., Fujimori, A., Taguchi, Y., Katsufuji, T., Tokura, Y., Europhys. Lett., 59, 258 (2002). 